\title{
Career Planning of Communication Engineering Students Hongli Zhu
}

\author{
School of Information and Electronic Engineering, Zhejiang University City College, \\ Hangzhou 310015, China \\ zhuhl@zucc.edu.cn
}

\begin{abstract}
With the development of teaching reform and demonstration construction in universities, the practice course of college students' career planning plays an increasingly important role in student education and teaching. It is helpful to improve the students' innovative ability and practical ability, to improve the teaching quality and realize the teaching goal in the vocational education practice teaching of college students. This paper shows the whole picture of the exploratory student activities in the planning design of university career and goal of this exploratory activity. We promote the ideological and political education of college students under the new situation.
\end{abstract}

Keywords: Career; Planning; College; Students

\section{通信专业的学生大学生涯规划}

\section{朱红丽}

( 浙江大学城市学院、信息与电气工程分院，浙江 杭州 310015)

摘要: 随着高校教学改革与示范建设的逐步推进, 大学生职业规划实践课程在学生教育教学工作中扮演着越来越重要的 作用。在高校学生教育教学工作中积极开展职业规划实践教学课程, 有利于提升学生的创新能力和实践能力, 提高教学质量, 实现教学目标。从大学生涯规划设计的意义、具体做法出发, 全面、系统地阐述了大学生涯规划设计这一探索性学生活动的 全貌, 揭示了这一探索性活动的目标, 为新形势下推动高校学生思想政治工作的创新提供借鉴和参考。

关键词: 职业; 规划; 大学; 学生

中图分类号：G712 文献标志码：A

\section{引言}

近年来，随著经济的快速成长，社会、经济、科技、文化、价值观的多变，对大学生而言，如何就业？ 如何成长? 如何生活? 如何学习? 如何谋生等都是叒待解决的问题。

生涯规划的价值在于厘清个人愿景，有效地认识自己，客观的认知环境与所需资源，并用合理可掌握 的方法, 逐步的将其整合, 以达成目标, 完成个人使命。

\section{1 大学生涯规划的重要性}

通信工程专业主要研究通信过程中信息传输和信号处理的原理和应用，需要学习通信技术、通信系统 和通信网等方面的知识。通信工程是电子工程的一个重要分支，电子信息类子专业，同时也是其中一个基 础学科。该学科是信息科学技术发展迅速并极具活力的一个领域, 尤其是数字移动通信、光纤通信、 Internet 网络通信使人们在传递信息和获得信息方面达到了前所未有的便捷程度。通信工程具有极广阔的 发展前景, 也是人才严重短缺的专业之一。

很多通信工程专业的大学新生是在完全不清楚本专业的就业前景和具体工作职责的状态下就选了专 业, 因此, 非常有必要在大学入学之初仔细思考, 规划好自己的大学生涯以及未来的职业生涯。一个懂得 
生涯规划意义的人，就是一个懂得自我发展规划的人，他会在每个发展阶段中，给予自己不同的塑造、训 练, 确认自己的志趣, 然后做好许多准备和良好习惯的养成。

\section{2 大学生涯及职业规划}

\section{1 钻研一门自己感兴趣的运动}

“健康来自健康的生活方式”，健康文明的生活方式是现代人应有的素质。所谓健康的生活方式，简 单地说就是人怎样健康地去生活。大学生是祖国的未来, 是国家宝贵的财富, 尤其在竞争日益激烈, 人才 国际化的今天, 对大学生的健康提出了更高的要求。

而通过自己感兴趣的运动还可以结识更多志同道合的朋友, 扩大自己的社交圈, 工作后也能够利用这 门运动来丰富自己的社交生活。同时, 有规律的运动可以帮助大学生建立良好的作息习惯, 有助于提高大 学课堂到课率和听课效果。

\section{2 学会常用的工具软件}

\section{2 .1 办公软件}

随着科学技术、社会经济的迅猛发展, 现代社会要求新时代的人才具有适应岗位、职业变化的能力。 以及计算机的普及办公的智能化, office 办公软件的学习变得越来越重要。

大学生需学会 Word 的全面运用，包括文书处理技巧；用页眉及页脚加入文件标题及页码；制作报价 单，会议议程及工作时间表; 加插图片、图表、文字艺术、制作图文并茂的文件或公司通讯; 不同资料大 量合拼打印; 精美文档的编辑、排版; 常用的、复杂的表格制作技巧等。

还需学会 Exce1 电子表格的处理、数据的运算管理、统计, 工资表、财务报表的制作, 用自定义功能 实现各种操作目标和个性化管理。学会综合运用各种 Excel 公式、函数解决复杂的管理问题和用 Excel 处 理及分析不同来源、不同类型的各种数据, 以及灵活运用 Excel 的各种功能进行财务数据分析和管理。

PowerPoint 是制作和演示幻灯片的软件，大学生应能够制作出集文字、图形、图像、声音以及视频剪 辑等多媒体元素于一体的演示文稿, 把自己所要表达的信息组织在一组图文并茂的画面中, 将来也可用于 介绍公司的产品、展示自己的成果。

\section{2.2 专业软件}

MATLAB 是一种在通信工程专业应用非常广泛的数据处理工具, 在"信号与系统"、“通信原理”、“数字 信号处理”等课程的实验教学中都会用到 MATLAB。学生在这些课程中学会使用 MATLAB 这个功能强大的仿真 软件, 可以更好地综合理解这几门主干课程的内容, 发挥自己的创造性和主观能动性, 能够很快学会借助于 仿真技术来分析和设计通信系统。

MATLAB 是美国 MathWorks 公司出品的商业数学软件, 用于算法开发、数据可视化、数据分析以及数值 计算的高级技术计算语言和交互式环境, MATLAB 可以进行矩阵运算、绘制函数和数据、实现算法、创建用 户界面、连接其他编程语言的程序等, 主要应用于工程计算、控制设计、信号处理与通讯、图像处理、信 号检测、金融建模设计与分析等领域。

\section{3 学好英语掌握通信前沿技术}

日常的查询中我们看到，作为互联网最大的网络百科全书----维基百科，涵盖的信息丰富、完整，具 有很大的参考价值。目前维基百科有超过 400 万条英语词条。而中文只有 70 万不到的词条。维基百科的 
Languages used on the Internet 词条中指出, 英语占互联网使用语言的 55\%。

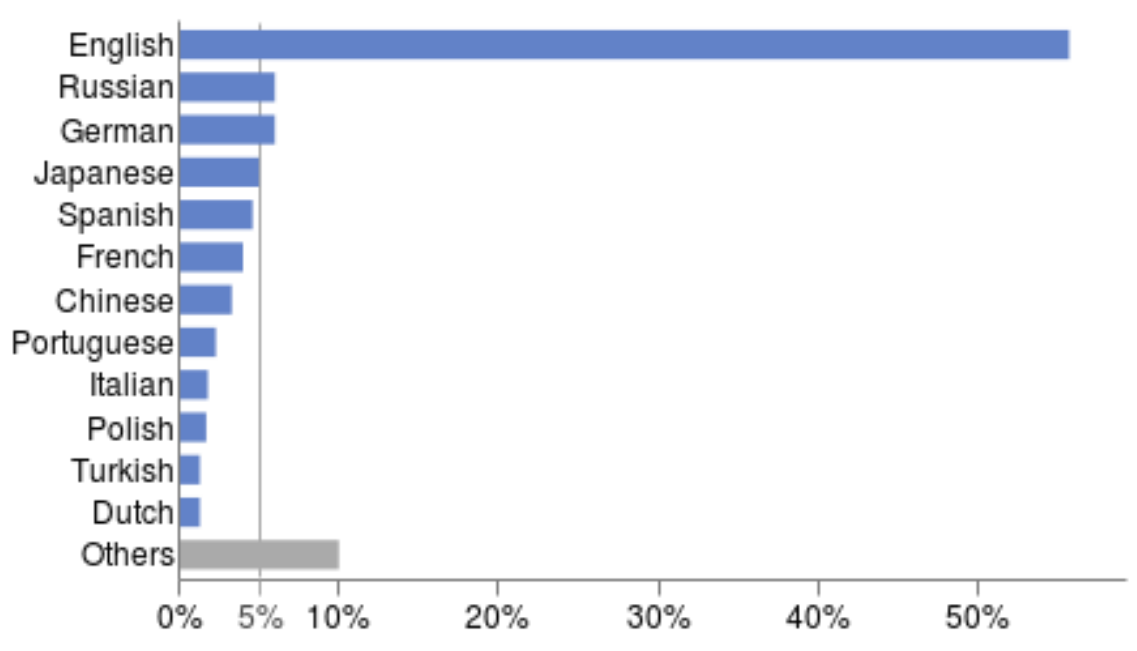

图 1 互联网语言覆盖图

因此, 从提高个人技能和竞争力来讲, 我们都有充分的理由学好英语。为了吸收通信技术前沿知识, 我们需要阅读很多科技文献, 而它们大多以英文的形式发表的, 很多的国际学术会议其通用语言也是英语。 为了获得一份理想的工作, 我们知道很多通信行业的公司对英语能力都有要求; 工作中遇到从国外引进的 技术资料需要我们有较强的英语阅读能力; 与境外客户沟通时需要较强的英语口语与写作能力。而有志于 继续深造的同学, 则会在做学术研究过程中每天都需要接触英语, 比如读英文文献、写英文论文、与国际 同行通过 EMAIL 和电话进行交流等。

除了提高专业技能外, 大学生还应该阅读非虚构类的书。这类书籍, 英文写的也不错, 知识也很实用, 有利于词汇量增加和阅读能力的精进。比如通信专业的同学有必要学习一下市场营销相关知识, 可以阅读 Philip Kotler 所著的 “Marketing Management: Application, Planning, Implementation and Control”, 该书对大学生了解市场营销的基本概念, 学习怎样制定营销战略、怎样抓住消费者的心理、怎样运用价格 争夺市场等方面有很好的价值。另外, 人力资源管理的英文版教材如 Gary Dessler 所著的“Human Resource Management” 详细介绍了人力资源管理的六大模块和日常事务、商业公司的大致构成, 看完后对大学生未 来就业和择业会有较好的指引。

\section{4 找三两份实习}

通信工程是一个很大的学科, 是一个理论与实践紧密结合的典型科目。通信工程学生所学颇杂, 就业 面广, 因此更需要尽早明确自己是适合做软件工程师, 或者适合做硬件开发, 还是适合做理论研究。要认 清自己的能力和兴趣所在, 最好的办法就是尝试, 大二、大三的时候就要开始实习。

大学生应学会写一份 HR 喜欢的简历, 参加几份商业领域的实习。争取换几个行业和职位, 多了解接 触以后个人的职业规划就慢慢形成了, 同时会明确的是学校的课程需要学什么以及怎么学。很多大学生对 课堂学习提不起兴趣, 原因之一在于他们不知道这门课的应用场景。通过实习, 他们会对各门课程有更清 晰的认知, 更珍惜大学生涯, 这对学风建设也是大有裨益的。

\section{3 结论}

大学生生涯规划的价值在于厘清个人愿景, 有效地认识自己, 客观的认知环境与所需资源, 并用合理 可掌握的方法, 逐步的将其整合, 以达成目标, 完成个人使命。本文从大学生涯规划设计的意义、具体做 
法出发, 全面、系统地阐述了大学生涯规划设计这一探索性学生活动的全貌, 揭示了这一探索性活动的目标, 为新形势下推动高校学生思想政治工作的创新提供借鉴和参考.

\section{References}

[1] Meng Xiu-juan, Yang Wen-juan: "Xiamen port competitiveness research based on factor analysis"[J]. Logistics Engineering and Management, Vol. 40(2010), No.9, p.52-55 (in Chinese)

[2] Zhang Jing-jing: "Southwest of port competitiveness evaluation based on factor analysis method" [J]. Logistics Engineering and Management, Vol. 31(2013), No.10, p.35-38 (in Chinese)

[3] Song Jian-ming, Wang Hai-yan: "Coastal ports quantitative evaluation method of economic contribution" [J]. China Water Transport, Vol. 52(2014), No.5, p.23-25 (in Chinese)

[4] Han Liang: Port economic externality research based on system dynamics (MS., Dalian Maritime University, China 2011), p.25-29.

[5] Zhang Xiao-yu: The interaction between port logistics and regional economic analysis (MS., Dalian Maritime University, China 2014), p.36-39.

[6] Huang Yao: The port logistics industry cluster and the relationship between economic development research (MS., Chongqing Technology and Business University, China 2014), p.29-35.

[7] Tang Miao-yan: "Try to talk about the value of the port logistics analysis" [J]. Manager Joumal , Vol. 66(2014), No.33, p.53-56 (in Chinese)

[8] Cai Shu-li: "The port logistics and economic growth relationship analysis" [J]. Joumal Changsha, University, Vol. 28(2015), No.02, p.76-79 (in Chinese)

[9] Information on http://baike.baidu.com

[10] Information on http://www.chinaports.com 